In the July issue the new Editor-in-Chief mentioned a number of ways in which it is hoped the Journal will develop. This process has started, but will not be noticeable until January. Meanwhile, this issue has two predominant themes: hormonal therapies and young people.

\section{HORMONE THERAPIES}

Hormones and breast cancer risk have been much in the news in the last few months, and Jo Marsden provides a commissioned commentary entitled Progestogens and breast cancer risk: the role of hormonal contraceptives and hormone replacement therapy (page 185). We also present the new Faculty Guidance on First Prescription of Combined Oral Contraception (page 209). Frequently asked questions looks at the use of the combined oral contraceptive pill in women taking enzyme-inducing medication (page 225).

\section{YOUNG PEOPLE}

Provision of services for young people is always under review, and there are several papers looking at important issues beginning with the editorial by Karen Rogstad and Helen King on Child protection issues and sexual health services in the UK (page 182) which gives an overview of the current situation and previews the forthcoming new Sexual Offences Reform Bill and the potential implications of the changes in legislation for health professionals.

Access by the unaccompanied under-16-year-old adolescent to general practice without parental consent A survey of primary health care providers in West Cornwall, UK studied the willingness of general practitioners (GPs) and practice nurses (PNs) to consult with under-16-year-olds without parental consent and asked about policies to facilitate access by unaccompanied under-16-year-olds (page 205). The majority of GPs and PNs were prepared to consult with unaccompanied under16-year-olds but many practices did not appear to have policies in place to guide health professionals and receptionists. This local survey is likely to be applicable to many areas of the UK and challenges us to consider the need for policies and staff training.

Barriers to the involvement of clients in family planning service development: lessons learnt from experience

A team from South London, UK demonstrate how clinic users can be trained to interview other clinic users to generate ideas to improve services from the client's perspective (page 199). Interestingly the staff group saw improving clinical services as most important whereas the client group saw the comfort and ambiance of the waiting room as equally important. The process of client consultation can help ensure that future service developments meet the priorities identified by both groups.

Promoting sexual health services to young men: findings from focus group discussions

The National Strategy for Sexual Health and HIV in the UK requires the provision of appropriate, accessible sexual health services for those who need them. Young men know less about available services and use them less frequently. The paper on page 194 looks through the eyes of focus groups around England at ways of improving accessibility to services for young men and highlights the need for initial contact with services by young men to be quick and straightforward and the importance of involving them in designing promotion campaigns. This paper needs to be read in conjunction with Men's use of sexual health services (page 190), which looks at data from a variety of sources to gain an insight into the use of sexual health services by men during the 1990s. Consistently men are less likely to use services that are seen as primarily providing contraception. Genitourinary medicine provides the only service where male and female use is approximately equal. Both papers highlight the need for more research in this important area.

\section{PREDICTING PROBLEMS WITH IUD INSERTION}

Intrauterine device insertion-related complications: can they be predicted?

This useful clinical paper (page 227) reports on a retrospective case note analysis to review the incidence of complications experienced at the time of inserting an intrauterine device (IUD) and up to 12 weeks later. The paper shows that IUD insertion-related complications are linked to anatomical and physiological factors, with nulliparous women experiencing more fitting complications. The experience of the doctor is also important in reducing complications. Postcoital IUD insertions were no different to planned IUD fittings.

\section{THE ROLE OF ACUPUNCTURE}

A review of controlled trials of acupuncture for women's reproductive health care

Acupuncture is being used increasingly in primary and secondary care. An understanding of its uses will provide health professionals with the information to advise patients appropriately. This review article on page 233 describes the different approaches used by various practitioners and possible mechanisms of action. It reviews the controlled trials of the effectiveness of acupuncture and allied techniques such as acupressure in women's reproductive health.

\section{EVIDENCE-BASED MEDICINE}

Our evidence-based medicine series continues with a look at the evidence for diagnostic tests, taking the tests for the management of postmenopausal bleeding as the example (page 245).

\section{HIV AND INFERTILITY}

Infertility treatment for HIV-positive couples: far from immaculate conception

The Margaret Jackson Prize Essay 2003 (page 239) considers the emotive issue of infertility treatment when one or both partners are known to be HIV-positive.

\section{MEDICAL TERMINATION OF PREGNANCY}

\section{Medical termination in the late first trimester}

The winner of the Organon Laboratories Award for the best oral presentation at the Faculty of Family Planning and Reproductive Health Care AGM presents an interesting paper on the place of medical termination as an option after 63 days as currently covered by the license for early medical abortion (page 243).

Anne Szarewski, Fran Reader and Beccy Hobbs For the Editorial Team, Journal of Family Planning and Reproductive Health Care 